\section{The first green revolution}

\author{
IshengJ. Tsai
}

This month's Genome Watch describes how analysis of a basal member of the Plantae can inform us about the evolution of photosynthetic eukaryotes.

Ask someone what makes humans different from plants and the first answer will most probably be the process of photosynthesis. However, photosynthesis is not limited to plants but is also undertaken by bacterial species as well as other eukaryotes, such as algae. The theory of primary endosymbiosis describes how the ancestors of plants acquired a photosynthetic capability by engulfing a free-living cyanobacterium that carried out photosynthesis, the genome of which was reduced over time to leave a functional organelle - known as a plastid - that depends on proteins encoded in the nuclear genome of the eukaryotic host. Plantae is a taxonomic supergroup of eukaryotes that comprises the green and red algae, the land plants and the glaucophytes (microscopic photosynthetic freshwater algae). Previous analysis of the plastid genome sequences of diverse members of the Plantae revealed that they form a monophyletic group (monophyly), which suggests that the ancestor of the plastids in the whole Plantae group was a single cyanobacterial species ${ }^{1}$.

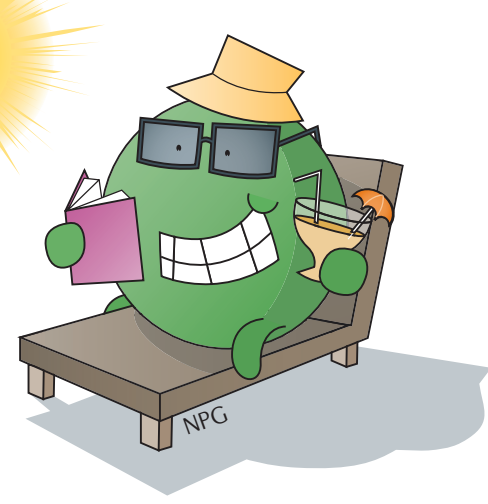

However, the question remains as to how many times the eukaryotic ancestor (or ancestors) took up the cyanobacterial plastid ancestor. To help resolve the origin of the Plantae group and the plastids that it contains, Price et al. have produced an assembly of $\sim 70 \mathrm{Mb}$ from the nuclear genome of the glaucophyte Cyanophora paradoxa CCMP329 (REF. 2). The glaucophytes are of particular interest because the common assumption is that they emerged prior to the divergence of green plants and red algae. Furthermore, glaucophyte plastids are unique in that the bacterial peptidoglycan layer is still present between their two membranes, placing them at an intermediate stage between endosymbiont and organelle ${ }^{3}$.

From the genome assembly and transcriptomic data, 27,921 genes were predicted, of which $\sim 17 \%$ are orthologous to published bacterial, archaeal and/or eukaryotic genes. Using this sequence data, the authors constructed 4,445 phylogenetic trees, which provided evidence of the different origins of C. paradoxa genes. Phylogenetic analyses showed that $\sim 60 \%$ of the genes in the C. paradoxa genome with orthologous relationships support a sister group relationship between glaucophytes and red and green algae, and presumably were present in the eukaryotic ancestor of the Plantae. The origins of $6 \%$ of the genes were found to be cyanobacterial; some of the proteins encoded by these genes contain transit sequences for plastid import, suggesting that the genes arose by endosymbiont gene transfer from the plastid ancestor to the host nucleus. One example of such a gene is that encoding fructose-1,6-bisphosphatase; the C. paradoxa genome contains the plastidderived gene rather than a gene encoding the cytosolic form that is seen in other species. An origin in bacteria other than the cyanobacterial plastid precursor was suggested for $10 \%$ of the genes, possibly as a result of horizontal gene transfer (HGT).
The authors also searched through the predicted genes of $C$. paradoxa for landmark traits of a typical photosynthetic eukaryote. For example, the authors identified a minimal set of putatively cyanobacterium-derived translocon subunits in the C. paradoxa genome, indicating that the plastid protein translocation system was present in the single Plantae ancestor. Interestingly, when searching for sugar phosphate transporters that are needed for transport of carbon metabolites between the host cell and plastid, instead of the expected plastid phosphate translocator, the authors identified two UhpC-type hexose phosphate transporters that are related to those found in Chlamydia spp., suggesting that genes involved in plastid function were also acquired from other bacteria through HGT events.

By analysing a sequenced algal genome at the basal branch of the Plantae phylogeny, this work has revealed new details about the origin of plastids. Further analysis of the genomes of basal species in the Plantae will enable us to characterize genes from the common ancestor, identify early features of the photosynthesis system and pinpoint the different genes that plants gained as they became more specialized.

\section{Isheng J. Tsai is at the Sanger Institute, Wellcome Trust Genome Campus, Hinxton, Cambridge CB10 1SA, UK. e-mail:microbes@sanger.ac.uk doi: 10.1038/nrmicro2781}

1. Hedges, S. B. et al. A molecular timescale of eukaryote evolution and the rise of complex multicellular life. BMC Evol. Biol. 4, 2 (2004).

2. Price, D. C. et al. Cyanophora paradoxa genome elucidates origin of photosynthesis in algae and plants. Science 335, 843-847 (2012).

3. Pfanzagi, B. et al. Primary structure of cyanelle peptidoglycan of Cyanophora paradoxa: a prokaryotic cell wall as part of an organelle envelope. J. Bacteriol. 178, 332-339 (1996).

Competing interests statement

The author declares no competing financial interests. 